Volume 8. No. 10, October 2020

International Journal of Emerging Trends in Engineering Research

Available Online at http://www.warse.org/IJETER/static/pdf/file/ijeter1458102020.pdf

https://doi.org/10.30534/ijeter/2020/1458102020

\title{
A Review of Multi-objective Evolutionary Algorithms for Information Retrieval System
}

\author{
Rama Gaikwad ${ }^{1}$, Ramanathan $L^{2}$ \\ ${ }^{1}$ Reasearch Scholar, School of Computer Science \& Engineering, VIT, Vellore, India, rama.coer@ gmail.com \\ ${ }^{2}$ Associate Professor, School of Computer Science \& Engineering, VIT, Vellore, India, lramanathan@ vit.ac.in,
}

\begin{abstract}
Information retrieval systems (IRSs) presentation is generally calculated using two special principles, accuracy and analysis. Exactness is the proportion of appropriate documents retrieved by the Information Retrieval System to the overall number of archives retrieved in the context of a client's inquiry; while review is the proportion of the quantity of pertinent reports recovered to the complete number of important reports for the client's question that are present in the narrative database. We present an investigation of two notable broadly useful multi-objective transformative algorithms, Challenges of Multi-objective Evolutionary Algorithms, Applications and Recent improvements in Multi-objective Evolutionary Algorithms all briefly. Further this examination talks about the diverse writing attempts to make the examination.
\end{abstract}

Key words : Query learning, Information retrieval systems; Multi-objective evolutionary algorithms

\section{INTRODUCTION}

Multi-objective optimization problems (MOPs) are normal. Think about the idea for a complex hardware / programming interface, for example, because it is being capable of to be used in mobile phones, cars, etc. without much stretch. The expenditure of these systems is to be reduced as much as possible, while most extraordinary execution is required. Other objectives, such as steadfast quality and dissipation of power, could be critical relying on the application. They can either be explicitly depicted as discrete movement models or distinguished as goals, for example, that the size of the framework must not outperform determined measurements. This paper provides an audit of the Multi-objective Evolutionary Algorithms for Information Retrieval System.

\section{Multi-obJective Evolutionary Algorithms}

Over as long as three decades, Evolutionary Algorithms (EA s) have amply demonstrated their pledge in taking charge of numerous search and improvement problems. One of EA 's strengths and specialties is their willingness to tackle multi-target improvement problems in their entirety, which their old style partners demand. Recommended in the early 1990s, transformative multi-target streamlining (EMO) algorithms are currently routinely used to address issues with numerous conflicting destinations in various parts of construction, science, and commerce. Two moderately comparative sorts of issues can be separated in unwinding a MOP (Horn 1997): journey and dynamic. The key perspective insinuates the movement procedure where the doable assortment for Pareto Optimal structures is. Also with a solitary item advancement, colossal and complex inquiry spaces can make search troublesome and hinder the utilization of cautious improvement procedures, for example, straight programming (Steuer 1986). The accompanying conclusion talks about the issue of choosing from the Pareto-perfect bundle for a reasonable exchange off plan. Five of the most striking MOEAs have been picked for explicit studies.

\subsection{Schaffer's Vector Evaluated Genetic Algorithm [74]}

Schaffer introduced a MOEA known as Vector Assessed Hereditary Calculation (VEGA), a representative of class assurance through trading goals. Here, for each of the goals k, choice is made independently, filling comparatively evaluated pieces of the mating pool.

\subsection{Hajela and Lin's Weighting-based Genetic Algorithm[75]}

It relies on the weighting technique, and the heaps are not set but rather encoded in the individual's vector to scan in equal measure for specific course of action. With respect to a potentially remarkable weight mix, each person is evaluated in this way.

\subsection{Fonseca and Fleming's Multi-objective Genetic Algorithm [76]}

Fonseca and Fleming suggested a Pareto-based location methodology (here the shortened FFGA type is used where an individual's role is equivalent to the whole Population-encoded system in which the inclination variable relates is overwhelmed absolutely. 


\subsection{Horn, Nafpliotis, and Goldberg's Niched Pareto Genetic Algorithm [77]}

The genetic algorithm (GA is gladly tailored to deal with several objectives by incorporating the notion of Pareto dominance in its selection operator, The Niched Pareto GA as an algorithm for finding the Pareto optimal set. We demonstrate its ability to find and maintain a diverse "Pareto optimal population" on two artificial problems and an open problem in hydrosystems

\subsection{Srinivas and Deb's Nondominated Sorting Genetic Algorithm [82]}

Among the Pareto based MOEAs, Srinivas and Deb performed Goldberg's sketch in the simplest manner possible. The complex frontal interaction of the population are, metaphorically, bit by bit stripped off and wellness sharing is performed independently for each front to ensure decent variety.

\section{Key Challenges of Multi-objective EVOLUTIONARY ALgORITHMS}

Multi-objective optimization problems (MOPs) are normal. For example, think about the structure of an unpredictable programming / equipment framework, as it can be found very well in cell phones, vehicles, and so on. The expense of such frameworks is often to be limited, whereas most extreme execution is sought. A classification of the general procedures that manage these problems is introduced in the accompanying. The emphasis here is on a pleasant look through the populace where there is only one enhancement run to estimate the Pareto Optimal set. In addition, another problem, elitism, is addressed easily, as it is special and more consistent with MOPs than with SOPs.

- Fitness Assignment and Selection

Instead of a single-target advancement, where target capacity and wellness work are regularly indistinguishable, a few destinations with MOPs must be taken into account in both the wellness task and the choice. All in all, MOEAs can be recognized where the objectives are deemed independent, approaches that depend on the traditional collection procedures, and strategies which utilize the idea of Pareto predominance.

\section{- Population Diversity}

Developmental streamlining agents ought to carry out a multimodal hunt where multiple, typically separate structures are to be identified to incorrect the Pareto-ideal collection in a single enhancement race.

Hence, maintaining a different population is significant to a MOEA's viability. Shockingly, a straightforward (elitist) EA generally unites toward a solitary arrangement and regularly loses arrangements due to three impacts): pressure of determination, commotion of choice, and disturbance of service operators.

- Elitism
De Jong [78] proposed a technique to fuse Pt's best individual reliably into $\mathrm{Pt}+1$ in order to prevent misfortune due to interruption of the effect test or service operator. This method, which can be spread out for the individuals to come to copy the best $\mathrm{b}$ responses, is inferred as exclusiveness. In his analyses, De Jong found that exclusiveness can improve the presentation of a hereditary calculation on uni-modal capacities.

\section{SOLVING TEChNiQues For Multi-ObJECTIVE Problems}

Multi-objective optimization problems resolved using GAMS programming were designed to provide the option to recognize the effects of the fuse of monetary and natural factors over ideal structure and conditions of operation in layer falls for protein fractionation of hydrolysate.

\section{APPLICATIONS AND RECENT DEVELOPMENTS IN Multi-obJeCtive Evolutionary Algorithms}

The use of multi-objective evolutionary algorithms (MOEAs) in research field is, currently, still quite insufficient, essentially when compared with regard to the use of single-objective evolutionary algorithms. So the most important purpose of this paper is specifically to catch the attention of the attention of EMOO researchers towards this field, which is very capable and contains a diversity of motivating and demanding problems. The use of MOEAs in construction has been very broad. Building technologies thus include a broad variety of problems, such as the construction of welded frames, bulk carriers, airfoil, new enticing devices, enhancement of groundwater monitoring systems, combinatorial logical loops, self-regulating navigation cars, control mechanisms, polymer expulsion concerns, development assistance,city and provincial arranging, covering visit issue, directing and supersonic wings. The MOEA provides some curved subsets of the arrangement of each workable portfolio and, subsequently, a basic line algorithm is illuminated for each subset. In the long run, the halfway agreements are converged in order to obtain the response for the first non-raised question.

\section{INFORMATION RETRIEVAL SYSTEMS}

Information retrieval is the move to get data assets from an assortment of data assets that are applicable to a data need. It is a piece of data science which investigates those exercises which identify with recovering data. Searches are mostly focused on metadata and also on full-content indexing Element retrieval element:

Information Retrieval primarily composed of four elements, i.e.

a. Information carrier.

b. Descriptor.

c. Document address.

d. Transmission of information. 
Information carrier: Carrier is something that has something to carry, to hold or to convey. In the other side, the information carrier seems to be a device that holds or stores data. For example: CDs, DVDs, Floppy Disks, Magnetic Tapes etc

Descriptor: The phrase or terminology used to check for capability data is classified as Descriptor. It's recognized as the watchwords that we use from a power gadget to scan info. Document address: Each record must have a location that differentiates that archive area.

Transmission of Information: When required, the transmission of information intends to gracefully record any record due to the clients. To do this, the data recovery framework uses different channels of correspondence or system management apparatuses.

The fundamental elements of the framework for Information Retrieval are to gracefully correct data at a perfect time due to the right client. Information Retrieval Framework elements are discussed below as follows:

Acquisition: It is the primary and main knowledge retrieval function. Securing intends to gather data from a variety of sources. Right off the bat, the company collects their perfect data from a number of outlets. Sources could be Book, Report, Database, Diary, and so on.

Contest analysis: The second step of the framework for information recovery is to break down their data obtained, and in this progress they may choose whether or not this report they collect is important.

Content presentation: Exchange of information is a system for the communication of information to the customer. The information is to be explained in such a way that it could be easily understood by the customer.

For this reason inventory, list of sources, list, and current mindfulness administration will be helpful.

Creation of file/store: The library authority makes another record at this stage to store their collected data, which is being prepared for introduction. They sort those documents out into some sort of organized method.

Creation of search methods: The authority chooses at this stage what kind of quest rationale they can use to search and recover data.

Dissemination: The last phase of Recovery Framework for Information is spread. It is the demonstration that data is generally spread. The library authority disperses data in an orderly fashion to the client at the stage.

\section{LITERATURE REVIEW}

The Review of Literature will discuss the related work of the study.

Czajkowski and Kretowski, [25] discuss the multi-objective design approach to model-tree recognition. The model tree is a specific instance of a tree of preference built to take care of relapse problems. Given the fact that the selection tree enlistment is innately a multi-objective task, the greater part of daily learning estimates may only be done a solitary target that may potentially total different objectives. Tests conducted demonstrate that the solution is prepared to discover Pareto front for the GMT framework. This counts as an initial step towards looking for effective and easy Pareto front for relapse and model trees that are to be accustomed based on consumer inclinations.

Cui et al. [2] study how to boost the analysis of what is more, adequacy of the estimation of the plan by utilizing the multi-target improvement method. Two goal capacities are shown, and another topic marker of various variety is placed. It is mainly used to gage the computation capacity of the suggestion to suggest distinctive theme types of things. And then we suggest a multi-objective probabilistic enhancement calculation (called PMOEA). Cremene et al. [38] intend to analyze several other multi-target estimates, especially those focused on DE.A different examination line considers smart EMOAs : instead of registering the Pareto Set and choosing a posteriori the preferred arrangements, the chief inclinations are prepared at run time. The algorithm of the search must focus on a single piece of the Pareto Collection along such lines, cor-reacting to the inclinations of the chief and reducing the computational cost henceforth.

Coello [57] presents a diagram of the most critical research work conducted on the subject of the arrangement of computationally extensive multi-object optimization problems. The methodologies that are examined quickly incorporate problem guess, work estimate (i.e., proxies) and transformative estimate (i.e., legacy of bunching and wellness). Coello [55]offers a brief overview of a portion of the main points where disruptive multi-target changes are being explored by flow. The topics discussed incorporating new calculations, productivity, loosened strength types, adaptability and meta-heuristics options.

In Cheshmehgaz,Haron and Sharifi,[51] the related works in the territory of utilizing different developmental calculations to look for ideal arrangements of multi-target streamlining issues are reviewed. With the these days equal and appropriated figuring frameworks, the pattern in utilizing increasingly viable numerous ventures expounds the ongoing patterns of various MOEAs; notwithstanding, the greater part of these calculations significantly depend on the isolating strategies and neighborhood transformative pursuits that are not proficient and successful.

A multi-objective transformative method named MOPA is suggested in Cheng et al. [21] to streamline the pAUC within a discretionary positive false range. Under this context, it is suggested that two important divisions focus on a single district within the AUC.Initial, another measurement (K-FPR), is recommended that is made by thinking about the fractional scope of the bogus positive rate (FPR). This is joined with the genuine positive rate (TPR) to give the two improved goals of MOPA.

Cheng et al . [65] will explore the application of large-scale knowledge analysis and EC algorithms, for example, the use 
of EC algorithms, to resolve large-scale information evaluation problems and the use of information investigative techniques to prepare new EC algorithms, or to enhance the display of EC algorithms. In the light of the mix of EC algorithms and information processing techniques, one can see better the bits of information inquiry expertise, and layout progressively qualified algorithms to possibly understand certifiable big issues of information analysis.

Cecchini et al. [39]provides an organized analysis of the different analytical methods intended for handling the problem of assorted variety survival in theme-based shooting, and presents a clear illustration of the Big Data use. The methodology to understanding topical issues and the methodologies for achieving decent variety are thoroughly optimized and can be functional to recover data identified with any subject for which a depiction and a lot of important archives (preparing set) are accessible.

Cao et al. [42] proposes an equally agreeable co evolutionary multi-objective developmental calculation (DPCCMOEA) based Message Passing Interface (MPI). DPCCMOEA handles deterioratingly dependent MOLSOPs. To begin with,one can we divide preference variables into a few assemblies, each of which is strengthened by a subpopulation (animal groups), in view of a modified element review technique.Cano et al.[31] presents a multi-objective hereditary programming measurement centered in Pareto to illustrate the abstraction and the representation of knowledge. The measurement is designed to achieve improvements in details that facilitate the implementation of structures and interpretations of both modified and imbalanced knowledge. Six order and representation measures are distinguished as targets to be upgraded by the multi-target calculation.

Bechikh, Elarbi and Ben Said, [43] have first described the related issues experienced by MOEAs when the dimensionality of the target space increases. The target-decrease-based methodology finds the relevant destinations and dispenses with the excess targets which are not essential to portray the ideal Pareto front.The inclination fuse based methodology abuses the inclinations of DM in order to separate proportionate arrangements between Pareto. It centers the hunting procedure around a given Pareto front district.

Bandyopadhyay [18] builds a professional prescient or enlightening model of a great deal of knowledge that suits or clarifies it better, yet may, on the other side, sum up fresh details. The simple knowledge set may be clear as numbers and characters, gradually mistaken as trees and tables, or even unstructured as text, depending on the application. Bandaru, $\mathrm{Ng}$ and Deb[47] deal with information mining techniques that can be applied to extricate knowledge from the arrangements generated during multi-object optimization produced during streamlining. This information is required to give further bits of knowledge about the issue to the leader, notwithstanding helping the advancement procedure in future structure cycles through a specialist framework.

In El Aziz, Ewees and Hassanien, [11] a new non-commanded system is suggested for content-based image recovery (NSMOWOA) contingent on multi-target whale streamlining estimation.The proposed technique keeps away from the disadvantages in other non-commanded arranging multi-target strategies that have been utilized for content-based picture recovery through lessening the reality intricacy.

Bhatnagar and Pareek,[19] proposes a joint coordinating capacity to improve the Information Retrieval System (IRS) productivity; The suggested structure uses an Evolutionary approach to change the loads of the research correlated with similitude. Ascia et al. [66] focus on framework-level structure, proposing a wide-ranging review of best-in - class MOEA approaches with a Fuzzy algorithm speed up an up-and-comer framework setup assessment. The analysis is carried out in a true concrete investigation: progression of the display and force distribution of embedded models in a compact digital software device room based on a Very Long Instruction Word (VLIW) chip.

A disintegration based transformative algorithm is presented in Asafuddoula, Ray and Sarker, [45], and its exhibition is shown using unconstrained and compelled numerous goal advancement problems. The algorithm is structured using a consistent state structure.Although seeking to lighten the scalarization-related problems (generally faced with comparison heading-based strategies), unity between respectable variety and union is preserved using a conspired simple preemptive separation association.A dynamic multi-objective, memory-improved transformative algorithm focused on depletion of Lp (indicated by dMOEA / D-Lp) is proposed in Xu et al . [16] . In specific, dMOEA / D-Lp decays and at the same time advances a complex multi-object optimization question into numerous efficient scalar advancement subproblems. The Lp deterioration strategy is alluded and altered to utilize.

Antonio and Coello [23] is a basic survey of the most active Coevolutionary Multi-Objective Evolutionary Algorithms (MOEAs) accounted for in the specific lettering. This study incorporates a scientific approach categorization along with a concise depiction of their core features. Antonelli, Ducange and Marcelloni,[61]focus on MOEAs introduced to adjust the normative foundation and the Mamdani FRBS framework concurrently and attempt to cope with the problem by exploiting the co-operative energy between two distinctive techniques. The first approach relies on a innovative method that decreases the pursuit room by introducing laws that are not from scratch;but rather from a heuristically produced rulebase.These cond method plays out an occasion determination by misusing a co-developmental methodology where consistently a genetic algorithm advances a diminished 
Rama Gaikwad et al., International Journal of Emerging Trends in Engineering Research, 8(10), October 2020, 7577 - 7588

preparing set which is utilized in the advancement of the MOEA.

Altwaijry and Menai,[30] have launched a review of the different information structures used in MOEAs to update both the document (non-commanded so-lutions) and the entire population. As various areas and sections of MOEAs this area was not generally and widely distributed. Aleti and Moser, [70] systematically research the best in the class for versatile control of parameters. The methodologies are clustered by another rational model that splits the way to modify algorithms of parameters into four phases that are unambiguously or definitely possible in a single current technique that effectively adjusts parameters throughout the improvement phase.

In Sathya and Suman,[20], crawler is used for social event and the elimination of data from the available documents from electronic repositories and different repositories. Given the enormous amount of search space, Genetic Algorithm ( GA) is used to discover the words of the combination.T he catchphrases are extricated from the record crawler in the proposed report recovery framework and with these watchwords GA produces a mix terms. Tamoor et al. [14] have presented a novel methodology by mapping the issue as a multi - objective optimizing issue to limit the number of significant code sections (classes) and boost the comparability between the bug report and the recovered piece of code. The arrangement depends on the closeness of a bug report with different classes to the cosine.

In Sharma and Virk, [66] ,several techniques were used to locate the ideal arrangement. Some of them manage an insightful choice of single object and some with multi objective. The equations using the pareto placed together technique dependent on predominance rating, power width, pareto intensity and so on to achieve the optimal multi-target scheme. Kalita and Gupta, [41] surveyed a few MOEAs used for rule mining associations. Significance was granted on the representation of the gene, goal capacities, disruptive managers and the dedication of the last structure. In addition, a correlation is given between different strategies in each classification.

Von Lücken, Barán and Brizuela [17] represents a survey of how the use of MOEAs in multi-object issues that depict the advancement of the field, the techniques that have been created, also the main discoveries and open inquiries that should be suggested in order to keep the field molding. Several approaches have been accounted for and summed up, even like the key successes of the trial and the practical experiments, to enable the curious peruser grasp the bleeding edge of the area as well as to find potential opportunities for study. Martín, Menéndez and Camacho1,[22] merge bunching and multi-objective optimization to create a classifier that is based on the specific activities of outsider call gatherings. The optimiser guarantees the recognition of such meetings of malignant or beneficial activities that clean up every non-discriminatory instances.

Golchin and Wee-Chung Liew, [26] first represent standard bi-bunch concepts that are commonly used. Then it is depicted a few groundbreaking bi-group formulas focused on their target capacities, search mechanisms and whether they support their performance. Finally, this study is reviewed take a shot at a bi-clustering algorithm depending on Pareto 's transformative front quality calculation called PBD-SPEA. We display usage of PBD-SPEA for details on content articulation, multimodal knowledge, for example, Big Data or image dataset, or Twitter or Facebook dataset.

In Zhao et al .,[13], this paper proposes a multi-objective, transformative intuitionist, fluffy bunching al-gorithm with various spatial image data to illuminate the division of images. This methodology uses the intuitive fluffy collection to resolve the weakness of photographs and, meanwhile, uses various spatial picture details to counteract the effect of photos clamour. Zhou et al.,[52] first structure a multi-target enhancement model for multiperiod dynamic emergency resource scheduling (ERS) issues. At that point, utilizing the system of multi-objective developmental c algorithm dependent on disintegration (MOEA/D), an MOEA is proposed to comprehend this model. In the said calculation, new transformative administrators are structured with the inborn properties of multi-period dynamic ERS issues at the top of the priority list.

Zhou et al. [58]represented a group of MaOEAs for many objective matters. Exploratory findings on 80 benchmark issues showed that by combining the positives of distinctive MaOEAs into one arrangement, EMaOEA not only offers professionals with a related framework to recognize their range of problems, but can also request preferred execution over a single MaOEA.Zhou et al. , [72] surveys the progress of MOEAs over the last few years, generally. It unwraps algorithmic systems, such as decay-based MOEAs (MOEA / Ds), memetic MOEAs, coevolutionary MOEAs, supervisors of choice and posterity propagation, MOEAs with explicit pursuit techniques, MOEAs for multimodal issues, limitation taking care of and MOEAs, computationally costly multiobjective streamlining issues (MOPs), dynamic MOPs, boisterous MOPs, combinatorial and discrete MOPs, benchmark issues, execution pointers, and its potential applications.

Zheng, Liu and Chen, [44] propose a revolutionary estimate to resolve multi-object optimization problems, called EAPD-RD, based on $\mathrm{M} 2 \mathrm{M}$ population decline and comparison separation. Upon demographic decline, the nation decays into a few sub-populations and each sub-community corresponds to a sub-culture referred to By a vector of course.

Zapotecas-Mart'inez et al. ,[56] present an evaluation of the features and confines of existing multi-target test problems defined in persistent and unconstrained pursuit spaces . In 
addition, a survey of suggestions and features for the structure of versatile test problems has been introduced. Fresh features have been applied to the catalog of dissecting products as an integral aspect of the dedication.

Yen and He, [53] introduce a Multi-Objective Developmental Estimates (MOEAs) collection technique by combining various implementation metrics utilizing dual-end rivalry alternatives. The double end configuration allows for distinctly dreadful display of an algorithm of quality to even now have the option of winning everything. Xu et al.[48] proposed a MOEA / HD, which layers subproblems into various progressions and adapts the inquiry heading for each subproblem of the lower-pecking order according to the progressive framework and the $\mathrm{Xu}$ et al. [16] examines systematically the use of EMO for multi-clustering (i.e. simultaneous scanning of numerous bunching).A powerful bi-target model is manufactured in which the quantity of clusters and the whole square distance(SSD) between information centers and their cluster centroids are considered destinations. A novel methodology of change is applied to the SSD to guarantee that the two destinations clash with each other. At that point, an EMO algorithm is used to fathom the model.

Wang et al. ,[7]present a hybrid multi-object Firefly Algorithm (HMOFA) for huge streamlining of the information. A number of broad knowledge streamlining problems are being evaluated in the trials, including six single aim problems and six multi-goal issues. Computational tests reveal that HMOFA performs promisingly on all research problems.Wang et al. , [8] make a proposed multi-target system for long-tail things. Within this system, two competing goal capacities are de-marked to reflect, separately, the capacities of advising mechanism to propose particular and unpleasant items. A novel, multi-objective transformative algorithm is proposed to streamline these two target works.Wang et al. , [54] seeks to enhance both the precision and reasonable diversity of these structures by utilizing developmental managers to identify sub-populations nearby. A neighborhood-based execution system is introduced in which a population is divided into a pre-characterized number of sub-populations using different leveled clustering .Condition choice and hereditary variety are then applied to every subpopulation.

Vikhar[64] provides a basic diagram of Evolutionary Algorithms and their non-exclusive usage technique. It further examines the different points of interest down to earth, using transformative algorithms for improvement over old style strategies. It additionally incorporates bizarre investigation of different invariants of EA like Genetic Programming (GP), Genetic Algorithm (GA),Evolutionary Programming (EP) and Evolution Strategies (ES). Vachhani, Dabhi and Prajapati, [73] examined the different strategies used by MOEAs to defend the varied variety structure. This study will be of value to learners in recognizing contrasts between various MOEAs. Often Pareto set includes a larger number of arrangements than file size, so raising the size of the Pareto set is important.

Trivedi et al. , [59] The extensive work analysis discussed takes a stab at MOEAs dependent on degradation. The study shows that numerous decay-based MOEAs have been proposed in writing since the recommendation of the first MOEA / D system in 2007. Soui et al. ,[4] proposed and rule-based methods for credit chance surveys. Utilizing characterization rules permits producing an exact, understandable, adaptable, and dynamic assessment model. The basic thought of this work is the creation of intriguing rules of order. This issue is regarded as a multi-target issue where the ultimate aim is to locate the best standards that limit chance, limit unpredictability and increase precision. Limiting danger is ensured with the proposed model being highly interpretable.

Srinivasan and Ramakrishnan, [67] reviews Evolutionary multi target enhancement (EMOO) frameworks mulling over the above measures. Other hybridization techniques include the use of canny experts, fuzzification, meta-information and meta-heuristics, parallelization, client experience, understanding, and so on, which further improve the display and ease of usage of the system. Segura et al.,[37] offers a refreshed review of the principle techniques that permit the utilization of multi-target plans for single-objective optimization. Multi Objectivization has been applied effectively to a few complex enhancement challenges. Multi-objectivized plans have been shown to give much preferable arrangements over comparative single-target plans for a few instances. Studies were conducted that depended on both experimental and hypothetical investigations.

Sanchita and Anindita, [3] discuss Big information and standard calculation restrictions dealing with them, Evolutionary Algorithm and their points of interest in taking care of Big information, Commonly used Evolutionary Calculation - Genetic Algorithm and the various application regions where hereditary Algorithm assumes developmental work in a vast and complex area of inquiry. Roitero et al. ,[6] build up an original and productive methodology based on a multi-objective transformative algorithm. The superior proficiency of their new execution allows us to mimic some remarkable results on the subject-set decrease, just as new tests are performed to summarize and progress such results.

Saada et al., [28] propose a robotized approach, in view of multi-target streamlining, to recuperate change follows between models. The approach takes a source model and its related objective model as the info. The point is to locate the many-to-many coordinating connections between the two models, partnering with a collection of $\mathrm{m}$ source components along those lines to a collection of $\mathrm{n}$ target components. Rey et al. ,[29] selected the most satisfactory guidelines for a superior accuracy-interpretability tradeoff as a genuine factor for the exchange of accuracy-interpretability off, for both 
dispersed and phonetic FRBSs. An examination of other announced methodologies indicated that rule pertinence was presented for this purpose.

Ravi, Pradeepkumar and Deb, [24] propose two 3-phase hybridhyubrid expectation models in which Chaos hypothesis is used to build stage space (Step-1) followed by taking the pairs Multi-Layer Perceptron (MLP) (Stage-2) and Multi-Objective Particle Swarm Optimization (MOPSO)/Elitist Non-Overpowered Sorting Genetic Algorithm (NSGA-II) (Stage-3). Stage-3 improves the gage yielded by stage- 2 in both of these cross-variety models. Ravber, Mernik and Crepinsek, [63] evaluated and dissected QIs with CRS4EAs inorder to comprehend their inclination better and to perceive how they can influence the positioning of MOEAs.Once QIs on five dif-ferent MOEAs, settling unconstrained CEC 2009benchmark MOPs, and a genuine issue called CITO, are being thought about. In MOES positioning, selected QIs were ordered into clusters which had inconsistent contrasts.

Qu et al. ,[36] surveys the cutting-edge research identified in that heading. It covers subjects of run of the mill MOEAs, old style EED issues, Dynamic EED issues, EED issues joining wind power, EED issues consolidating electric vehicles and EED issues inside miniaturized scale frameworks. What's more, some potential bearings for future research are likewise introduced.

Purshouse et al., [62] reviews strategies which have joined developmental multi-target streamlining and different measures dynamic. Three types of half breed approaches are introduced: a posteriori, from the earlier, and intuitive, including methods used to demonstrate the inclinations of the leaders and model algorithms for each individual.

Ponsich estimated time of arrival [68] has given the best in class study of use of MOEAs in financial matters and account detailed in the literature in question. The initial one managed the portfolio improvement issue and spoke to by far most of the work revealed in this area.

Pascual et al. [46] explore the utilization of MOEAs to create at runtime ideal designs of the DSPL as per diverse criteria.The improvement issue is formalized as far as a FeatureModel(FM), a inconstancy model. Onan, Korukoglu and Bulut, [12] set up a powerful notional order plot by seeking a worldview of the pruning ground forces. Collecting pruning is an essential technique for producing groups of classifiers with high prescient precision and skill. Exponential inquiry, randomized searching, successive pursuit, placement-based pruning, and bunching-based pruning were used in past study.

In Saini , Saha and Bhattacharyya, [10] a subjective enlivened multi-target programmed a report bunching method is proposed which is a combination of self-sorting out guide
(SOM) and multi-target differential development approach. The variable number of group focus is encoded in different populace arrangements to decide in a computerized manner the quantity of clusters from an informational collection. During progression these systems undergo different genetic practices.

Ojha , [71] covers difficulties related with MOEAs, diverse arrangement approaches, for example, Pareto-based techniques and non-Pareto strategies, pointer based methods, aggregation strategies, disintegration based techniques, techniques utilizing reference sets, MOEAs including DM, from the earlier, intelligent and a posteriori inclination joining techniques. It additionally talks about a large portion of the quality measurements and execution pointers proposed in the writing alongside benchmark issues. Mukhopadhyay et al. ,[9] build a prescient or illustrative, productive model of a lot of information. Uses of transformative algorithms were seen as particularly valuable for the programmed preparation of huge amounts of crude loud information for the ideal setting of parameters and for finding large and important data.

Muhammad Iqbal Bayumurti et al [79] summarizes the use of genetic algorithms for supply chain management and improves the efficiency of the same using ant colony optimization techniques. This study provide evidence that the GA can solve the supply chain optimization problems.

S. Nagendram et al., [80] talks about trending algorithms from machine learning which can help in optimizations They aim the Hadoop and ML amalgamation. The explanation of the Hadoop and the equivalent issues in case of the combined process of configuring the cluster has been outlined.

$\begin{array}{lrrr}\text { Mirjalili et } \quad \text { al., } & \text { [5] } & \text { proposes } \\ \text { Multi-ObjectiveGreyWolfOptimizer(MOGWO) } & \text { in }\end{array}$ request to enhance issues with various objects for the first time.A fixed-sized outer chronicle is coordinated to the GWO for sparing and recovering the Pareto Optimal Solutions. Maleki-Dizaji et al ., [32] addresses this problem with a flexible specialist put together demonstrating approach which depends on the demonstration of the developmental client. The proposed framework for recovery of data gained customer needs from the client gave important input.

Marcos-Pablos, Francisco and García-Peñalvo, [35] presents an iterative method that applies content mining and machine learning techniques to a downloaded corpus of updated works from conceptual repositories, consolidating programmed handling formulas with controlled dynamic tools in an iterative process accompanied by the decision of the scientists to modify, view and change the pursuit yields.

Metaxiotis and Liagkouras, [50] furnish a survey of the ebb and flow status of portfolio management research using Multi-Objective Evolutionary Algorithms (MOEAs). The use 
Rama Gaikwad et al., International Journal of Emerging Trends in Engineering Research, 8(10), October 2020, 7577 - 7588

of Multi-Objective Evolutionary Algorithms (MOEAs) for portfolio choice is still in its early stages. The investigation of the difficult's definition uncovered territories of worry with respect to MOEAs for the Portfolio Management.
Lupiani et al., [1] have moved toward the Case-Base Maintenance task as a multi-target advancement issue that might be explained with a Multi-Objective Evolutionary Algorithm. Specifically, the issue of advancement is divided into three extraordinary and concurrent objectives, including limiting the separation of the present number of cases for the

Table 1: Comparative Analysis

\begin{tabular}{|c|c|c|c|c|}
\hline Reference No & Method involved & Advantage & Disadvantage & Future scope \\
\hline 22 & $\begin{array}{l}\text { A method using the } \\
\text { third-party API } \\
\text { calls present in each Java file }\end{array}$ & $\begin{array}{l}\text { It provides improved } \\
\text { precision rates and } \\
\text { decreases the number of } \\
\text { false positives. }\end{array}$ & $\begin{array}{l}\text { The obfuscation } \\
\text { techniques act as } \\
\text { barriers. }\end{array}$ & $\begin{array}{l}\text { The accuracy needs to be } \\
\text { improved along with } \\
\text { approaches of big data. }\end{array}$ \\
\hline 41 & $\begin{array}{l}\text { MOEAs in Association } \\
\text { Rules }\end{array}$ & $\begin{array}{l}\text { The necessity to select a } \\
\text { single solution from the } \\
\text { non-dominated front is } \\
\text { not required. }\end{array}$ & $\begin{array}{l}\text { A large number of } \\
\text { itemsets along with } \\
\text { huge time is taken to } \\
\text { find all } \\
\text { Large itemsets. }\end{array}$ & $\begin{array}{l}\text { Computational } \\
\text { effectiveness of MOEAs } \\
\text { applied in association } \\
\text { rule mining tasks needs } \\
\text { improvement. }\end{array}$ \\
\hline 30 & $\begin{array}{l}\text { Linear lists, Quad } \\
\text { trees,Dominated trees }\end{array}$ & $\begin{array}{l}\text { Linear lists work well } \\
\text { for small population } \\
\text { problems.Quad trees } \\
\text { support larger } \\
\text { population problems. }\end{array}$ & $\begin{array}{l}\text { The performance gets } \\
\text { affected on controlling } \\
\text { the size and input } \\
\text { sequence. }\end{array}$ & $\begin{array}{l}\text { Statistical and under } \\
\text { standard test functions } \\
\text { need to investigate these } \\
\text { lists and trees. }\end{array}$ \\
\hline 61 & $\begin{array}{lrr}\text { A new approach for } & \text { for } \\
\text { supporting } & & \text { high } \\
\text { dimensional } & \text { and } & \text { large } \\
\text { datasets } & & \end{array}$ & $\begin{array}{l}\text { It supports } \\
\text { high-dimensional data. }\end{array}$ & $\begin{array}{l}\text { It does not support } \\
\text { low-dimensional data. }\end{array}$ & $\begin{array}{l}\text { Improvement is needed } \\
\text { in rule base complexity. }\end{array}$ \\
\hline
\end{tabular}

situation basis to an estimate of the measure of non-excess cases in the underlying case-base, reducing the extent of repetitive cases and limiting the estimate of the error rate attained with the case-base retained.

$\mathrm{Li}$ and Deb, [69] have examined the development of transformative multiobjective streamlining (EMO) issues with variable-length structures along the Pareto-ideal front. To draw out the difficulties looked by an EMO calculation, one have built five test issues for which the Pareto-ideal arrangements are established with various variable sizes.

Kishor,Singh and Prakash, [27] presents an on-commanded arranging based multi- objective fake honey bee state calculation SABC to explain multi-target advancement problems. It is an expansion of Artificial honey bee province calculation $\mathrm{ABC}$, which single target streamlining algorithm, to the multi-target enhancement domain. It utilizes a cover approach in the worker honey bee stage to guide the answers for at the same time accomplish both the symmetrical objectives the multi-target improvement - combination and assorted variety.

\section{Comparative ANALYSIS}

This section (Table 1) will comprise the comparative analysis of the different techniques in a tabular form. The comparison can be made with respect to the datasets being used, the objectives, the approach being used, results obtained, limitations etc.

\section{Conclusion}

This investigation showed how the Introduction of Information Retrival Systems (IRSs) is generally determined using two unusual standards, accuracy and verification. Precision is the extent of the significant reports recovered by the IRS in respect of the general number of classics recovered in relation to the customer's request; while review is the proportion of the quantity of pertinent reports recovered to the complete number of important reports for the client's question 
that exist in the narrative database. Thus an investigation of useful multi-objective transformative algorithms, Challenges of Multi-objective Evolutionary Algorithms, Applications and Recent improvements in Multi-objective Evolutionary Algorithms all briefly are discussed.Further this examination talks about the diverse writing attempts to make the examination.

\section{REFERENCES}

1. Lupiani, E., Massie, S., Craw, S., Juarez, J. M., \& Palma, J. (2016). Case-base maintenance with multi-objective evolutionary algorithms. Journal of intelligent information systems, 46(2), 259-284.

2. Cui, L., Ou, P., Fu, X., Wen, Z., \& Lu, N. (2017). A novel multi-objective evolutionary algorithm for recommendation systems. Journal of Parallel and Distributed Computing, 103, 53-63.

3. Sanchita, G., \& Anindita, D. (2016). Evolutionary algorithm based techniques to handle big data. In Techniques and Environments for Big Data Analysis (pp. 113-158). Springer, Cham.

4. Soui, M., Gasmi, I., Smiti, S., \& Ghédira, K. (2019). Rule-based credit risk assessment model using multi-objective evolutionary algorithms. Expert Systems With Applications, 126, 144-157.

5. Mirjalili, S., Saremi, S., Mirjalili, S. M., \& Coelho, L. D. S. (2016). Multi-objective grey wolf optimizer: a novel algorithm for multi-criterion optimization. Expert Systems with Applications, 47, 106-119.

6. Roitero, K., Soprano, M., Brunello, A., \& Mizzaro, S. (2018). Reproduce and improve: An evolutionary approach to select a few good topics for information retrieval evaluation. Journal of Data and Information Quality (JDIQ), 10(3), 1-21.

7. Wang, S., Gong, M., Li, H., \& Yang, J. (2016). Multi-objective optimization for long tail recommendation. Knowledge-Based Systems, 104, 145-155.

8. Wang, R., Lai, S., Wu, G., Xing, L., Wang, L., \& Ishibuchi, H. (2018). Multi-clustering via evolutionary multi-objective optimization. Information Sciences, 450, 128-140.

9. Mukhopadhyay, A., Maulik, U., Bandyopadhyay, S., \&Coello, C. A. C. (2014). A survey of multiobjective evolutionary algorithms for data mining: Part I. IEEE Transactions on Evolutionary Computation, 18(1), 4-19.

10. Saini, N., Saha, S., \& Bhattacharyya, P. (2019). Automatic scientific document clustering using self-organized multi-objective differential evolution. Cognitive Computation, 11(2), 271-293.

11. El Aziz, M. A., Ewees, A. A., \& Hassanien, A. E. (2018). Multi-objective whale optimization algorithm for content-based image retrieval. Multimedia tools and applications, 77(19), 26135-26172.
12. Onan, A., Korukoğlu, S., \& Bulut, H. (2017). A hybrid ensemble pruning approach based on consensus clustering and multi-objective evolutionary algorithm for sentiment classification. Information Processing \& Management, 53(4), 814-833.

13. Zhao, F., Liu, H., Fan, J., Chen, C. W., Lan, R., \& $\mathrm{Li}$, N. (2018). Intuitionistic fuzzy set approach to multi-objective evolutionary clustering with multiple spatial information for image segmentation. Neurocomputing, 312, 296-309.

14. Tamoor, M., Osama, S., Younas, I., \& Asif, S. Comparison of Different Multi Objective Evolutionary Algorithms for Bug Localization.

15. Wang, H., Wang, W., Cui, L., Sun, H., Zhao, J., Wang, Y., \& Xue, Y. (2018). A hybrid multi-objective firefly algorithm for big data optimization. Applied Soft Computing, 69, 806-815.

16. Xu, X., Tan, Y., Zheng, W., \& Li, S. (2018). Memory-Enhanced Dynamic Multi-Objective Evolutionary Algorithm Based on Lp Decomposition. Applied Sciences, 8(9), 1673.

17. Von Lücken, C., Barán, B., \& Brizuela, C. (2014). A survey on multi-objective evolutionary algorithms for many-objective problems. Computational optimization and applications, 58(3), 707-756.

18. Bandyopadhyay, S., Maulik, U., Coello, C. A. C., \& Pedrycz, W. (2014). Guest editorial: Special issue on advances in multiobjective evolutionary algorithms for data mining. IEEE Transactions on Evolutionary Computation, 18(1), 1-3.

19. Bhatnagar, P., \& Pareek, N. K. (2012). A combined matching function based evolutionary approach for development of adaptive information retrieval system. International Journal of Emerging Technology and Advanced Engineering, 2(6), 249-256.

20. Sathya, S. S., \& Simon, P. (2010). A document retrieval system with combination terms using genetic algorithm. International Journal of Computer and Electrical Engineering, 2(1), 1.

21. Cheng, F., Fu, G., Zhang, X., \& Qiu, J. (2019). Multi-objective evolutionary algorithm for optimizing the partial area under the ROC curve. Knowledge-Based Systems, 170, 61-69.

22. Martín, A., Menéndez, H. D., \& Camacho, D. (2017). MOCDroid: multi-objective evolutionary classifier for Android malware detection. Soft Computing, 21(24), 7405-7415.

23. Antonio, L. M., \& Coello, C. A. C. (2017). Coevolutionary multiobjective evolutionary algorithms: Survey of the state-of-the-art. IEEE Transactions on Evolutionary Computation, 22(6), 851-865.

24. Ravi, V., Pradeepkumar, D., \& Deb, K. (2017). Financial time series prediction using hybrids of chaos theory, multi-layer perceptron and 
multi-objective evolutionary algorithms. Swarm and Evolutionary Computation, 36, 136-149.

25. Czajkowski, M., \& Kretowski, M. (2019). A multi-objective evolutionary approach to Pareto-optimal model trees. Soft Computing, 23(5), 1423-1437.

26. Golchin, M., \& Liew, A. W. C. (2019). Bi-clustering by Multi-objective Evolutionary Algorithm for Multimodal Analytics and Big Data. In Multimodal Analytics for Next-Generation Big Data Technologies and Applications (pp. 125-150). Springer, Cham.

27. Kishor, A., Singh, P. K., \& Prakash, J. (2016). NSABC: Non-dominated sorting based multi-objective artificial bee colony algorithm and its application in data clustering. Neurocomputing, 216, 514-533.

28. Saada, H., Huchard, M., Nebut, C., \& Sahraoui, H. (2013, November). Recovering model transformation traces using multi-objective optimization. In 2013 28th IEEE/ACM International Conference on Automated Software Engineering (ASE) (pp. 688-693). IEEE.

29. Rey, M. I., Galende, M., Fuente, M. J., \& Sainz-Palmero, G. I. (2017). Multi-objective based Fuzzy Rule Based Systems (FRBSs) for trade-off improvement in accuracy and interpretability: A rule relevance point of view. Knowledge-Based Systems, 127, 67-84.

30. Altwaijry, N., \& Menai, M. E. B. (2012). Data structures in multi-objective evolutionary algorithms. Journal of Computer Science and Technology, 27(6), 1197-1210.

31. Cano, A., Ventura, S., \& Cios, K. J. (2017). Multi-objective genetic programming for feature extraction and data visualization. Soft Computing, 21(8), 2069-2089.

32. Maleki-Dizaji, S., Siddiqi, J., Soltan-Zadeh, Y., \& Rahman, F. (2014). Adaptive information retrieval system via modelling user behaviour. Journal of Ambient Intelligence and Humanized Computing, 5(1), 105-110.

33. Ye, Q. C. (2019). Multi-objective Optimization Methods for Allocation and Prediction (No. 2019-10).

34. Surya, S., \& Sumitra, P. Information Retrieval in Data Mining with Soft Computing Algorithms.

35. Marcos-Pablos, S., \& García-Peñalvo, F. J. (2018). Information retrieval methodology for aiding scientific database search. Soft Computing, 1-10.

36. Qu, B. Y., Zhu, Y. S., Jiao, Y. C., Wu, M. Y., Suganthan, P. N., \& Liang, J. J. (2018). A survey on multi-objective evolutionary algorithms for the solution of the environmental/economic dispatch problems. Swarm and Evolutionary Computation, $38,1-11$.
37. Segura, C., Coello, C. A. C., Miranda, G., \& León, C. (2016). Using multi-objective evolutionary algorithms for single-objective constrained and unconstrained optimization. Annals of Operations Research, 240(1), 217-250.

38. Cremene, M., Suciu, M., Pallez, D., \& Dumitrescu, D. (2016). Comparative analysis of multi-objective evolutionary algorithms for QoS-aware web service composition. Applied Soft Computing, 39, 124-139.

39. Baggio, C., Cecchini, R. L., Lorenzetti, C. M., \& Maguitman, A. G. (2016, November). An Entropy-Based Approach for Preserving Diversity in Evolutionary Topical Search. In Simposio Argentino de Inteligencia Artificial (ASAI 2016)-JAIIO 45 (Tres de Febrero, 2016).

40. Deb, K. (2015). Multi-objective evolutionary algorithms. In Springer handbook of computational intelligence (pp. 995-1015). Springer, Berlin, Heidelberg.

41. Kalita, M., \& Gupta, C. A Survey of Multi-objective Evolutionary Algorithms for Association Rule Mining.

42. Cao, B., Zhao, J., Lv, Z., \& Liu, X. (2017). A distributed parallel cooperative coevolutionary multiobjective evolutionary algorithm for large-scale optimization. IEEE Transactions on Industrial Informatics, 13(4), 2030-2038.

43. Bechikh, S., Elarbi, M., \& Said, L. B. (2017). Many-objective optimization using evolutionary algorithms: A survey. In Recent advances in evolutionary multi-objective optimization (pp. 105-137). Springer, Cham.

44. Zheng, Z., Liu, H. L., \& Chen, L. (2016, May). An evolutionary many-objective optimization algorithm based on population decomposition and reference distance. In 2016 Sixth International Conference on Information Science and Technology (ICIST) (pp. 388-393). IEEE.

45. Asafuddoula, M., Ray, T., \& Sarker, R. (2014). A decomposition-based evolutionary algorithm for many objective optimization. IEEE Transactions on Evolutionary Computation, 19(3), 445-460.

46. Pascual, G. G., Lopez-Herrejon, R. E., Pinto, M., Fuentes, L., \& Egyed, A. (2015). Applying multiobjective evolutionary algorithms to dynamic software product lines for reconfiguring mobile applications. Journal of Systems and Software, 103, 392-411.

47. Bandaru, S., Ng, A. H., \& Deb, K. (2017). Data mining methods for knowledge discovery in multi-objective optimization: Part A-Survey. Expert Systems with Applications, 70, 139-159.

48. Xu, H., Zeng, W., Zhang, D., \& Zeng, X. (2017). MOEA/HD: A multiobjective evolutionary algorithm based on hierarchical decomposition. IEEE transactions on cybernetics, 49(2), 517-526. 
Rama Gaikwad et al., International Journal of Emerging Trends in Engineering Research, 8(10), October 2020, 7577 - 7588

49. Cheng, S., Liu, B., Shi, Y., Jin, Y., \& Li, B. (2016, June). Evolutionary computation and big data: key challenges and future directions. In International conference on data mining and big data (pp. 3-14). Springer, Cham.

50. Metaxiotis, K., \& Liagkouras, K. (2012). Multiobjective evolutionary algorithms for portfolio management: A comprehensive literature review. Expert Systems with Applications, 39(14), 11685-11698.

51. Cheshmehgaz, H. R., Haron, H., \& Sharifi, A. (2015). The review of multiple evolutionary searches and multi-objective evolutionary algorithms. Artificial Intelligence Review, 43(3), 311-343.

52. Zhou, A., Qu, B. Y., Li, H., Zhao, S. Z., Suganthan, P. N., \& Zhang, Q. (2011). Multiobjective evolutionary algorithms: A survey of the state of the art. Swarm and Evolutionary Computation, 1(1), 32-49.

53. Yen, G. G., \& He, Z. (2013). Performance metric ensemble for multiobjective evolutionary algorithms. IEEE Transactions on Evolutionary Computation, 18(1), 131-144.

54. Wang, R., Fleming, P. J., \& Purshouse, R. C. (2014). General framework for localised multi-objective evolutionary algorithms. Information sciences, 258, 29-53.

55. Coello, C. A. C. (2009). Evolutionary multi-objective optimization: some current research trends and topics that remain to be explored. Frontiers of Computer Science in China, 3(1), 18-30.

56. Zapotecas-Martínez, S., Coello, C. A. C., Aguirre, H. E., \& Tanaka, K. (2018). A review of features and limitations of existing scalable multiobjective test suites. IEEE Transactions on Evolutionary Computation, 23(1), 130-142.

57. Coello, C. A. C. (2015). Multi-objective evolutionary algorithms in real-world applications: some recent results and current challenges. In Advances in evolutionary and deterministic methods for design, optimization and control in engineering and sciences (pp. 3-18). Springer, Cham.

58. Zhou, Y., Liu, J., Zhang, Y., \& Gan, X. (2017). A multi-objective evolutionary algorithm for multi-period dynamic emergency resource scheduling problems. Transportation Research Part E: Logistics and Transportation Review, 99, 77-95.

59. Trivedi, A., Srinivasan, D., Sanyal, K., \& Ghosh, A. (2016). A survey of multiobjective evolutionary algorithms based on decomposition. IEEE Transactions on Evolutionary Computation, 21(3), 440-462.

60. Bezerra, L. C., López-Ibáñez, M., \& Stützle, T. (2015, March). Comparing decomposition-based and automatically component-wise designed multi-objective evolutionary algorithms. In
International Conference on Evolutionary Multi-Criterion Optimization (pp. 396-410). Springer, Cham.

61. Antonelli, M., Ducange, P., \& Marcelloni, F. (2013). An efficient multi-objective evolutionary fuzzy system for regression problems. International Journal of Approximate Reasoning, 54(9), 1434-1451.

62. Purshouse, R. C., Deb, K., Mansor, M. M., Mostaghim, S., \& Wang, R. (2014, July). A review of hybrid evolutionary multiple criteria decision making methods. In 2014 IEEE congress on evolutionary computation (CEC) (pp. 1147-1154). IEEE.

63. Ravber, M., Mernik, M., \& Črepinšek, M. (2017). The impact of quality indicators on the rating of multi-objective evolutionary algorithms. Applied Soft Computing, 55, 265-275.

64. Vikhar, P. A. (2016, December). Evolutionary algorithms: A critical review and its future prospects. In 2016 International conference on global trends in signal processing, information computing and communication (ICGTSPICC) (pp. 261-265). IEEE.

65. Sharma, M. S., \& Virk, R. S. (2014). A review towards evolutionary multiobjective optimization algorithms. An International Journal of Engineering Sciences, Vol13/37-Vol13, 3.

66. Ascia, G., Catania, V., Di Nuovo, A. G., Palesi, M., \& Patti, D. (2011). Performance evaluation of efficient multi-objective evolutionary algorithms for design space exploration of embedded computer systems. Applied Soft Computing, 11(1), 382-398.

67. Srinivasan, S., \& Ramakrishnan, S. (2011). Evolutionary multi objective optimization for rule mining: a review. Artificial Intelligence Review, 36(3), 205.

68. Ponsich, A., Jaimes, A. L., \& Coello, C. A. C. (2012). A survey on multiobjective evolutionary algorithms for the solution of the portfolio optimization problem and other finance and economics applications. IEEE Transactions on Evolutionary Computation, 17(3), 321-344.

69. Li, H., \& Deb, K. (2017, June). Challenges for evolutionary multiobjective optimization algorithms in solving variable-length problems. In 2017 IEEE Congress on Evolutionary Computation (CEC) (pp. 2217-2224). IEEE.

70. Aleti, A., \& Moser, I. (2016). A systematic literature review of adaptive parameter control methods for evolutionary algorithms. ACM Computing Surveys (CSUR), 49(3), 1-35.

71. Ojha, M., Singh, K. P., Chakraborty, P., \& Verma, S. (2019). A review of multi-objective optimisation and decision making using evolutionary algorithms. International Journal of Bio-Inspired Computation, 14(2), 69-84. 
Rama Gaikwad et al., International Journal of Emerging Trends in Engineering Research, 8(10), October 2020, 7577 - 7588

72. Zhou, Y., Wang, J., Chen, J., Gao, S., \& Teng, L. (2017). Ensemble of many-objective evolutionary algorithms for many-objective problems. Soft Computing, 21(9), 2407-2419.

73. Vachhani, V. L., Dabhi, V. K., \& Prajapati, H. B. (2015, March). Survey of multi objective evolutionary algorithms. In 2015 International Conference on Circuits, Power and Computing Technologies [ICCPCT-2015] (pp. 1-9). IEEE.

74. Schaffer, J.. (1985). Multiple Objective Optimization with Vector Evaluated Genetic Algorithms.. Proceedings of the First Int. Conference on Genetic Algortihms, Ed. G.J.E Grefensette, J.J. Lawrence Erlbraum. 93-100.

75. Hajela P., Lee E., Lin CY. (1993) Genetic Algorithms in Structural Topology Optimization. In: Bendsøe M.P., Soares C.A.M. (eds) Topology Design of Structures. NATO ASI Series (Series E: Applied Sciences), vol 227. Springer, Dordrecht. https://doi.org/10.1007/978-94-011-1804-0_10

76. Fonseca, Carlos \& Fleming, Peter. (1999). Genetic Algorithms for Multiobjective Optimization: Formulation Discussion and Generalization. the fifth Intl conference on Genetic Algorithms. 93.

77. Horn, Jeffrey \& Nafpliotis, N. \& Goldberg, D.E.. (1994). A Niched Pareto Genetic Algorithm for Multi-Objective Optimization. Proceedings of the 1st IEEE Conference on Computation Evolutionary. 1. 82 - 87 vol.1. 10.1109/ICEC.1994.350037.

78. De J ong, K.A.: An Analysis of the Behavior of a Class of Genetic Adaptive Systems. Ph.D Thesis, University of Michigan (1975)

79. Muhammad Iqbal Bayumurti et al., Optimization of Goods Delivery in Supply Chain using Genetic Algorithm International Journal of Emerging Trends in Engineering Research, 8(9), September 2020, 5111 - 5115

80. S. Nagendram et al., Trending Algorithms in Machine Learning and issues along with Big data Context in real time Data Processing International J ournal of Emerging Trends in Engineering Research, 8(9), September 2020, 5760 - 5763

81. Amita Dhankhar et al., A Comprehensive Review of Tools \& Techniques for Big Data Analytics International Journal of Emerging Trends in Engineering Research, 7(11), November 2019, 556 - 562557

82. Srinivas, Nidamarthi, and Kalyanmoy Deb. "Muiltiobjective optimization using nondominated sorting in genetic algorithms." Evolutionary computation 2.3 (1994): 221-248. 